\title{
Neu-p11 induces NLRP3 inflammasome-induced pyroptosis via provoking NOX4-mediated mitochondrial oxidative injury in chordoma cells
}

\author{
Shutian Zhang $^{1 *}$ \\ ${ }^{1}$ Clinical Medicine (Eight-year Program), Shanghai Medical College, Fudan University, Shanghai \\ 200032, P.R. China
}

Correspondence should be addressed to: Shutian Zhang, MD.

E-mail: stzhang15@fudan.edu.cn

\begin{abstract}
Chordoma is a rarely malignant bone tumor with highly resistance to radiotherapy and chemotherapy. Melatonin has been shown to inhibit tumor cell invasion, metastasis and induce apoptosis in several types of cancer. Activation of melatonin receptor inhibit cancer stemness of chordoma, which suggesting that melatonin receptor agonists have potential therapeutic value for the clinical treatment of chordoma. The present study aimed to investigate the anticancer action and molecular mechanism of Piromelatine (Neu-P11) in chordoma cells, a high-efficacy agonist of melatonin receptor-1/melatonin receptor-2 (MT1/MT2). We usedSeu-p11 $(1,10,100,1000 \mu \mathrm{M})$ or vehicle to treat chordoma cell lines U-CHA land MUG-Chor for 36 hours. CCK-8 assay and LDH activity assay showed that Neu-p11 had dose-dependent cytotoxic effect on chordoma cells. Neu-p11 induced NLRP3 and Cleaved-Caspase-1 expression, while Caspase-1 specific inhibitor Z-YVAD-FMK and NLRP3 specific inhibitor MCC950 independently blocked the cytotoxic effect of Neu-p11 on chordoma cells, indicating that Neu-p11 induced a NLRP3-dependent cell pyroptosis. Neu-p11 promoted NADPH oxidase 4 (NOX4) translocation to mitochondria, resulting in a significant increase in mitochondria-derived ROS and decreased expressions of mitochondrial antioxidant proteins (MnSOD, Sirt3) and mitochondrial DNA replication factor (Tfam), which could be attenuated by NOX4 specific inhibitor GKT137831. Further investigation revealed that Neu-p11 resulted in decreased copy number of mtDNA and increased mtDNA releasing in cytoplasm. Decreased oxygen consumption, reduced membrane potential and ultrastructural damage of mitochondrial were detected in Neu-p11 treated chordoma cells, which could be attenuated by GKT137831. GKT137831 inhibited mitochondria-translocation of NLRP3 and attenuated activation of NLRP3 inflammasome caused by Neu-p11 treatment. Collectively, we demonstrated that Neup11 induces mitochondrial translocation of NOX4 leads to oxidative damage of mitochondria, mediating chordoma cells NLRP3-dependent pyroptosis. Neu-p11 may be a a new strategy for chordoma treatment.
\end{abstract}

Key words: Neu-p11, NOX4, Mitochondrial oxidative injury, NLRP3, Pyroptosis 


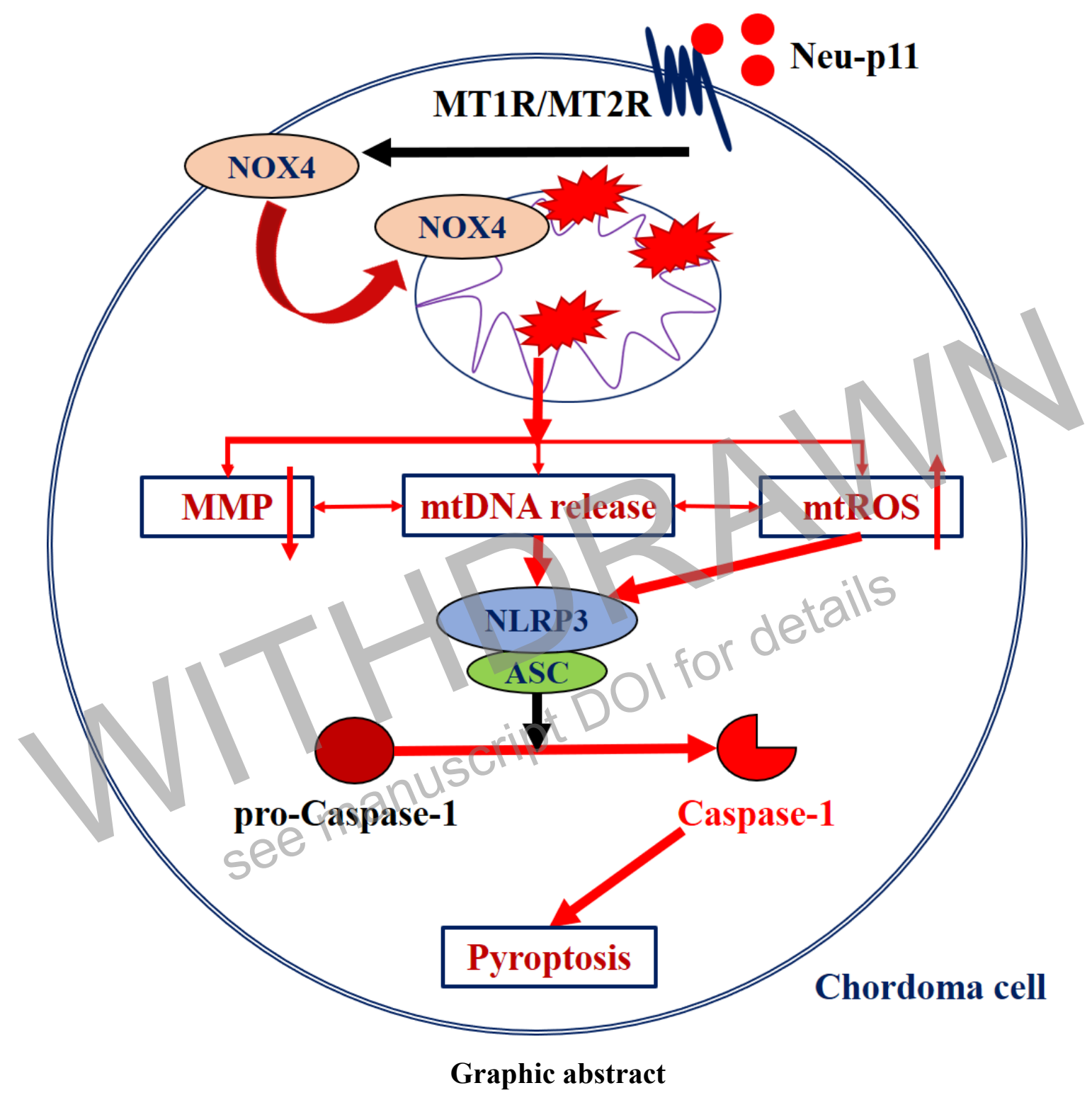


bioRxiv preprint doi: https://doi.org/10.1101/2020.02.12.946665; this version posted February 13, 2020. The copyright holder for this preprint (which was not certified by peer review) is the author/funder. All rights reserved. No reuse allowed without permission.

Abbreviations

Neu-p11, Piromelatine; NOX4, NADPH oxidase 4; NLRP3, NLR Family Pyrin Domain Containing 3; MnSOD, Manganese superoxide dismutase; Sirt3, NADdependent deacetylase sirtuin-3; Tram, mitochondrial transcription factor A; mAROS, mitochondria-derived reactive oxygen species; mitochondrial membrane potential (IMP, $\Delta \Psi \mathrm{m})$; mtDNA, mitochondrial DNA. 


\section{Introduction}

Chordoma is a primary malignant bone tumor, commonly occurring in the skull base, spine and sacrum. Chordomas are generally considered to have a slow growth rate but strong local invasion capability[1]. Chordoma is difficult to operate, the sensitivity to chemo-radiotherapy is very poor, the recurrence rate after treatment is very high[2]. Currently, no newly emerging cancer therapies such as targeted therapy and immunotherapy have been approved for chordoma treatment[3]. It is urgent to explore the biological characteristics of chordoma cells and find new therapeutic schemes.

Pyroptosis is a programmed cell death dependent on activation of Caspase-1, a conserved mechanism for maintaining cell homeostasis[4]. Pyroptosis was first studied in intracellular infection, and later proved to be widely involved in neurodegenerative diseases, autoimmune diseases, metabolic disorders, tumor development and other pathophysiological processes [5]. Induced cell pyroptosis is an important idea in tumor therapy[6]. Activation of intracellular pattern recognition receptors represented by Nucleotide binding oligomerization (NOD)-like receptors (NLRP3) is an important upstream event of Caspase-1 activation[7]. This pathway can be activated by a variety of endogenous and exogenous risk signals in the body including hypoxia, metabolic stress, calcium overload, and intracytoplasmic DNA, inducing the release of inflammatory cytokines and inducing cell death[8-10]. After tumor cells are subjected to multiple pressures such as oxidative stress, metabolic pressure and immune stimulation, mitochondrial dysfunction occurs, mtROS is produced, mitochondrial DNA (mtDNA) is oxidized and released into the cytoplasm, and finally NLRP3 is activated. This process is also accompanied by cardiolipin transport to the outer membrane of the mitochondria, promoting the attachment of NLRP3 to the mitochondria, and is believed to be involved in the activation of NLRP3[11, 12]. The role of mitochondrial damage and NLRP3 inflammasome-dependent cell pyroptosis in chordoma cells needs to be further elucidated.

Melatonin, an endogenous cancer suppressor hormone, has been shown to inhibit tumor cell invasion, metastasis and induce apoptosis in several cancer species[13, 14]. Recent studies have shown that melatonin is not only secreted rhythmically by the 
pineal gland, but also can be synthesized by peripheral tissue cells, regulating the redox balance and microenvironmental immunity of target cells by paracrine or autocrine patern[15, 16]. Numbers of relevant melatonin-dependent effects are triggered by targeting mitochondrial functions, including modulating the mitochondrial respiratory chain, antagonizing the highly glycolytic bioenergetic pathway, regulating $\mathrm{Ca}^{2+}$ release and mitochondrial apoptotic effectors[17]. The latest research results show that Activation of melatonin receptor inhibit cancer stemness of chordoma[18], which suggesting that melatonin receptor agonists have potential therapeutic value for the clinical treatment of chordoma.

Although most studies have demonstrated the antioxidant capacity of melatonin and its derivatives $[19,20]$, some have found that therapeutic concentrations of melatonin promote the production of reactive oxygen species (ROS) in tumor cells[21, 22]. Therefore, melatonin may be an "antioxidant" that has a conditioned pro-oxidant effect on tumor cells. The difference of melatonin in regulating redox homeostasis between normal cells and cancer cells suggests that melatonin can be used to kill chordoma cells specifically. However, little is known about the molecular mechanism by which melatonin promotes oxidative stress in tumor cells. There are two possible pro-oxidation mechanisms: melatonin interacts with NADPH oxidase (NOX) in tumor cells to stimulate ROS production; Melatonin can regulate mitochondrial complex III or mitochondrial permeability transition pore activity and promote the generation of mitochondrial ROS (mtROS). Mitochondria are the center of cellular redox regulation, and their functional and structural damage may be one of the mechanisms by which melatonin promotes oxidation to kill tumor cells. Melatonin can accurately regulate mitochondrial structural and functional balance and redox homeostasis[23, 24], block abnormal metabolic pathways of tumor cells and mitochondrial dependent signal transduction, and thus play a role in cancer inhibition. In addition, it can trigger mitochondria-dependent cell death in tumor cells.

Neu-p11, as a novel melatonin receptor agonist, has the advantages of longer halflife, higher selectivity, less adverse reactions and easy synthesis compared with melatonin[25, 26], which has great potential for clinical application. In this study, we 
bioRxiv preprint dol: https://doi.org/10.1101/2020.02.12.946665; this version posted February 13, 2020. The copyright holder for this preprint (which was not certified by peer review) is the author/funder. All rights reserved. No reuse allowed without permission.

found that Neu-p11 triggers mitochondrial translocation of NOX4 in U-CH1 and MChr chordoma cells, leading to mitochondrial oxidative damage and mediating NLRP3 inflammasome-dependent cell pyroptosis in chordoma cells. Neu-p11mediated proptosis may be a new strategy for the treatment of chordoma. 


\section{Methods}

\subsection{Drugs and reagents}

Neu-p11 (Piromelatine) was purchased from Neurim Pharmaceuticals Ltd. (Tel-Aviv, Israel); MCC950 (sc-505904) was purchased from Santa Cruz Biotechnology (Santa Cruz, CA, United States). Z-YVAD-FMK (235429) was purchased from Sigma-Aldrich (Sigma, St Louis, MO, USA). GKT137831 (B4763) was purchased from ApexBio Technology (ApexBio, Houston, Texas, USA). Oxygen Consumption Rate Assay Kit (ab197243) was purchased from Abcam (Cambridge, UK). Mito-SOX Red Mitochondrial Superoxide Indicator) (M36008) was purchased from Invitrogen (Carlsbad, CA, USA). Cell Counting Kit-8 (C0037), LDH Cytotoxicity Assay Kit (C0016), Mitochondrial membrane potential assay kit with JC-1 (C2006), Caspase 1 activity assay kit (C1101), Cell Mitochondria Isolation Kit (C3601) and Mitochondria Storage Buffer (C3609) were purchased from Beyotime (Nanjing, PRC). The antibodies used in this study are: $\beta$-actin (ab179467), NOX4 (ab133303), NLRP3 (ab232401), ASC (ab155970), pro-Caspase-1 (ab179515), PARP (ab74290), MnSOD (ab13533), Sirt3 (ab223531), Tfam (ab131607), COX IV (ab33985), Donkey Anti-Goat IgG H\&L(Cy5) (ab6566), Donkey Anti-Rabbit IgG H\&L(HRP) (ab6802) were purchased from Abcam (Cambridge, UK). Cleaved-Caspase-1(D57A2) was purchased from Cell Signaling Technology (Beverly, MA, USA); pro-IL-1 $\beta$ (AF-401) was purchased from R\&D Systems (Minneapolis, USA).

\subsection{Cell culture}

U-CH1 and MUG-Chor chordoma cells were purchased from the cell bank of the Chinese academy of sciences. Cells were grown in Eagle's minimum essential medium containing 10\% heat-inactivated fetal bovine serum (FBS) (Invitrogen, Carlsbad, CA), $100 \mathrm{U} / \mathrm{ml}$ of penicillin and $100 \mu \mathrm{g} / \mathrm{ml}$ of streptomycin in a humidified atmosphere of $5 \%$ $\mathrm{CO}_{2}$ at $37^{\circ} \mathrm{C}$.

\subsection{Cell viability assay}

Cell viability was measured in different treatment groups with Cell Mitochondria Isolation Kit (Beyotime) and preserved in Mitochondria Storage Buffer (Beyotime) according to the manufacturer's protocol. 


\subsection{Isolation of mitochondria}

Mitochondria from cells in different treatment groups were isolated with Cell Counting Kit-8 (Beyotime) and LDH Cytotoxicity Assay Kit (Beyotime) according to the manufacturer's protocol.

\subsection{Western blotting assay}

Total proteins were extracted from chordoma cells following immunoblotting as we previous described. $\beta$-actin was used as the control of cell lysate protein. COX IV was used as the control of mitochondrial lysate protein.

\subsection{RNA isolation and qPCR analysis}

Total RNA was isolated from microglia using TRIzol reagent (Invitrogen) according to the manufacturer's instructions. cDNA was transcribed and SYBR-Green-based real time quantitative PCR was performed according to the standard protocol.

\subsection{Transmission electron microscopy (TEM) study}

The cells were immobilized by $2.5 \%$ glutaraldehyde at $4{ }^{\circ} \mathrm{C}$ for $8 \mathrm{~h}$. TEM sections were made, observed and photographed by technicist from Electron microscopy Department, School of Basic Medicine, Fudan University.

\subsection{Oxygen Consumption Rate Assay}

Cell oxygen consumption rate was measured in different treatment groups with Oxygen Consumption Rate Assay Kit (Abcam) according to the manufacturer's protocol.

\subsection{Immunofluorescent staining}

Immunofluorescence technique was used according to the standard protocol.

\subsection{Mitochondria-derived ROS detection}

Mitochondria-derived ROS was determined by staining microglia with Mito-SOX Red Mitochondrial Superoxide Indicator (Invitrogen) according to the manufacturer's protocol.

\subsection{Caspase-1 activity assay}

Caspase-1 activity was measured in different treatment groups with Caspase 1 activity assay kit (Beyotime) according to the manufacturer's protocol.

\subsection{Detection of mtDNA copy number and cytoplasmic DNA content}

This part of the experimental steps refer to Bai J et. al.[37] 
bioRxiv preprint dol: https://doi.org/10.1101/2020.02.12.946665; this version posted February 13, 2020. The copyright holder for this preprint (which was not certified by peer review) is the author/funder. All rights reserved. No reuse allowed without permission.

2.13 Statistical analyses

All data are presented as mean \pm standard error of the mean (SEM). For experiments that involved two groups of samples, Student's unpaired t test was used. For experiments that involved multiple groups, one-way or two-way analysis of variance with repeated measures were used to assess group means. 


\section{Results}

\subsection{Neu-p11 inhibits cell viability and activates NLRP3 inflammasome on} chordoma cells

First, CCK-8 kit and LDH cytotoxicity kit were used to detect the effect of Neu-p11 treatment at different concentrations for $36 \mathrm{~h}$ on the viability of chordoma cells (Fig.1 A-D). We found that the viability of chordoma cells treated with $1 \mu \mathrm{M}$ Neu-p11 decreased compared with the control group, and the difference was statistically significant $(\mathrm{n}=5, * P<0.05)$. With the increase of Neu-p11 concentration in cell culture medium (1-1000 $\mu \mathrm{M})$, cytotoxicity has been significantly enhanced, and is concentration-dependent with Neu-p11 concentration $(\mathrm{n}=5, * P<0.05)$. We detected the protein expression of each component of NLRP3 inflammasome (NLRP3, ASC, pro-Caspase-1, Cleaved-Caspase-1 and pro-IL-1 3 ) by western blot, and found that Neup11 significantly increased the protein expression of each component of NLRP3 inflammasome (Fig. 1 E-I, $\mathrm{n}=5, * P<0.05$ ). The protein expression of each component of NLRP3 (NLRP3,ASC, pro-Caspase-1, Cleaved-Caspase-1 and pro-IL-1 $\beta$ ) increased with the concentration of Neu-p11 in the cell culture medium $(1-1000 \mu \mathrm{M})$, and was dose dependent on the concentration of Neu-p11 $(\mathrm{n}=5, * P<0.05)$. These results showed that Neu-p11 inhibited the viability of chordoma cells and activated the NLRP3 inflammasomes.

\subsection{Neu-p11 induces NLRP3-dependent pyroptosis on chordoma cells}

Pyroptosis is a programmed cell death dependent on Caspase-1 activation. In order to investigate whether Neu-p11 $(1000 \mu \mathrm{M})$ treatment induced pyroptosis in chordoma cells, we added the Caspase-1 specific inhibitor Z-YVAD-FMK into the cell culture medium, and found that Z-YVAD-FMK could partially block the cytotoxic effect caused by Neu-p11 (Fig.2 A, B, n = 5, $* P<0.05$ ), proving that Neu-p11 triggered the pyroptosis pathway of chordoma cells. Caspase-1, a component of the NLRP3 inflammasome, could be activated NLRP3. To further define whether Neu-p11 activates Caspase-1 through NLRP3-dependent manner, we added the NLRP3 specific inhibitor MCC950 into the cell culture medium, and found that MCC950 did not affect the expression of pro-Caspase1, but inhibited the increased expression of Cleaved- 
Caspase-1 caused by Neu-p11 treatment, indicating that Neu-p11 promoted the maturation of Caspase-1 by activating NLRP3 (Fig.2 C-F, $\mathrm{n}=5,{ }^{*} P<0.05$ ). We also found that MCC950 can inhibit the expression of DNA-damage-responseor PARP in chordoma cells caused by Neu-p11 (Fig.2 C-F, $\mathrm{n}=5, * P<0.05$ ), further suggesting that the pyroptosis of cells caused byNneu-p11 is dependent on the activation of NLRP3.

\subsection{The activation of NOX4 by Neu-p11 causes oxidative damage to the mitochondria of chordoma cells}

ROS in cells are mainly produced by NADPH oxidase and mitochondrial electron respiration chain. Recent studies have found that NOX4 could translocate from cell membrane to mitochondria, mediate mitochondrial oxidative damage, and is a key molecular bridge connecting the two ROS-generating systems. To investigate the effect of Neu-p11 $(1000 \mu \mathrm{M})$ on redox homeostasis in chordoma cells, we examined the effect of Neu-p11 at different concentrations onNOX4 expression in chordoma cells. We were pleasantly surprised to find that Neu-p11 significantly increased the expression of NOX4 at concentrations of $10-1000 \mu \mathrm{M}$ (Fig.3 A-C, $\mathrm{n}=5, * P<0.05)$. In order to further investigate whether Neu-p11-activated NOX4 affect mitochondrial redox state, we added the NOX4 specific inhibitors GKT137831 into the cell culture medium, found that GKT137831 inhibit the expression reduction of mitochondrial antioxidant proteins (MnSOD, Sirt3) and mitochondrial DNA replication factor (Tfam) in chordoma caused by Neu-p11 (Fig.4 A-E, $\mathrm{n}=5,{ }^{*} P<0.05$ ), suggesting that Neu-p11-activated NOX4 mediates the reduction of mitochondrial antioxidant capacity and damage of mitochondrial function in chordoma cells. To further verify this finding, we used MitoSOX mtROS indicator to observe the effect of GKT137831 on mtROS in chordoma cells treated with Neu-p11, and found that GKT137831 significantly reduced the accumulation of mtROS in chordoma cells induced by Neu-p11 (Fig.5 A-C, $\mathrm{n}=5,{ }^{*} P$ $<0.05$, Scale bar $=30 \mu \mathrm{m})$. We also detected mtDNA copy number and cytoplasmic mtDNA content of chordoma cells with reference to Bai J et. al.[37], and found that GKT137831 significantly reduced the mtDNA copy number and cytoplasmic mtDNA content of chordoma cells treated with Neu-p11 (Fig.6 A-D, $\mathrm{n}=5, * P<0.05$ ). Further detection of oxygen consumption rate (Fig.6 E-F, $\mathrm{n}=5, * P<0.05$ ) and mitochondrial 
membrane potential (Fig.7 A-C, $\mathrm{n}=5, * P<0.05$, Scale bar $=50 \mu \mathrm{m}$ ) confirmed that inhibition of NOX4 could block mitochondrial damage in chordoma cells caused by Neu-p11. We also used Transmission electron microscopy to observe the ultrastructural damage of mitochondria in different treatment groups, and confirmed that GKT137831 could block mitochondria structure damage of chordoma cells caused by Neu-p11 (mitochondrial swelling, mitochondrial intima structure blurring, and mitochondrial crest disorders) (Fig.8, Scale bar $=2 \mu \mathrm{m})$. These results suggest that Neu-p11-mediated NOX4 activation causes mitochondrial oxidative damage in chordoma cells.

\subsection{NOX4 inhibitor GKT137831 inhibits Neu-p11-mediated NOX4 mitochondrial} translocation and NLRP3 co-localization with mitochondria in chordoma cells

To determine whether mitochondrial translocation has occurred in Neu-p11-activated NOX4, we isolated the mitochondria of chordoma cells in different treatment groups and detected the expression of NOX4 in the mitochondria. As expected, Neu-p11 (1000 $\mu \mathrm{M}$ ) triggers the mitochondrial translocation of NOX4, which is inhibited by the NOX4 inhibitor GKT137831. We also examined the expression of NLRP3 on mitochondria, and found that Neu-p11 promoted mitochondrial translocation of NLRP3, while NOX4 inhibitor GKT137831 inhibited mitochondrial translocation of NLRP3, suggesting that NLRP3 could perceive mitochondrial damage caused by NOX4 activation (Fig.9 A-D, $\mathrm{n}=5, * P<0.05)$.

\subsection{NOX4 inhibitor GKT137831 inhibits Neu-p11-mediated pyroptosis of chordoma cells}

We further examined the effect of NOX4 inhibitor GKT137831 on the activation of NLRP3 inflammasome and found that GKT137831 inhibited Neu-p11-mediated activation of NLRP3 inflammasome (Fig.10 A-D, $\mathrm{n}=5, * P<0.05)$. Further, we found that GKT137831 inhibited the expression of the Neu-p11-mediated DNA damage responsor PARP (Fig.10 A, B, E, $\mathrm{n}=5, * P<0.05$ ), blocking the Neu-p11-induced chordoma cell viability impairment (Fig.10 F, G, $\mathrm{n}=5, * P<0.05$ ) and Caspase-1 activity (Fig.10 H, I, $\mathrm{n}=5, * P<0.05$ ). These results suggest that Neu-p11 leads to NLRP3 inflammasome-dependent pyroptosis of chordoma cells by activating NOX4. 


\section{Discussion}

For a long time, the treatment of chordoma has been in the dilemma of drug availability for a long time, partly because the incidence of chordoma is low, and mostly sporadic cases, and the medical community has paid relatively limited attention to it, resulting in a far less understanding of the biology of chordoma. Chordomas tend to occur in the spine and clivus, adjacent to the spinal cord and brain, so it is important to explore a new treatment that selectively kills chordoma cells and reduces damage to normal cells in adjacent areas[1-3]. In this study, we found that Neu-p11, a novel melatonin receptor agonist, was effective in inducing NLRP3 inflammasome-dependent apoptosis of chordoma cells. The upstream molecular events that trigger NLRP3 activation are NOX4 activation and mitochondrial translocation mediated mitochondrial oxidative damage. In addition, we noted that NOX4 activation was significantly activated only at relatively high concentrations of Neu-pjl (100-1000 $\mu \mathrm{M})$, while at relatively low concentrations of Neu-p11 $(1-10 \mu \mathrm{M})$ the activity was antichordoma. This suggests that the anti-tumor effect of Neu-p11 does not depend solely on the Nox4-mitochondriaNLRP3 inflammasome-Caspase-1 pathway. Our results not only provide experimental evidence support for the application of melatonin receptor agonists represented by Neup11 in the treatment of chordoma, but also provide new thinking for the understanding of the biological characteristics of redox homeostasis in chordoma.

The redox homeostasis of cells can be adapted to different metabolic and functional states. NOXs and mitochondrial electron transport chain produce ROS to play the role of intracellular messengers to regulate energy metabolism, while mitochondria and other organelles rich in antioxidant system can protect cells by removing ROS through dismutase such as MnSOD[27], suggesting that the redox homeostasis of cells comes from the coordination of several redox modulating systems. For a long time, it was thought that NOXs only existed in mononucleus-macrophages and neutrophils for killing pathogenic bacteria, while ROS in tumor cells mainly came from mitochondrial electron transport chain. In fact, NOXs gene expression and its dynamic regulation are very active in a variety of tumor cells $[28,29]$. This gives us a new anticancer idea: whether we can activate NOXs in tumor cells, which causes tumor cells to suffer from 
ROS overload, and ultimately inhibit the proliferation and invasion of tumor cells. To our surprise, our study found that the use of Neu-p11 to stimulate the melatonin receptor in chordoma cells can effectively activate NOX4 and ultimately kill the chordoma cells. This provides experimental evidence to support the anticancer idea.

In general, melatonin is considered a classic antioxidant molecule. Most studies on the new medicinal uses and mechanisms of melatonin have found that melatonin can play an antioxidant role through the following two pathways: receptor-dependent signal transduction pathway to activate the antioxidant system[30, 31]; Or nonreceptordependent mechanism as the antioxidant substrates directly neutralize oxidants[32]. Most studies have tended to suggest that melatonin plays a protective role by downregulating the expression of NOXs in normal cells [33]. Melatonin may have no significant effect on the expression of NOXs in tumon cells $[34,35]$. We found that the melatonin receptor agonist Neu-p11 can activate NOX4 mitochondrial translocation in chordoma cells and cause oxidative stress. Our findings are consistent with previous reports that melatonin produces ROS through mitochondrial complex III[21]. We interpret this result as the genetic molecular phenotypes of tumor cells and normal cells are very different from each other, resulting in the abnormal linkage between the downstream signaling pathway of melatonin receptor and NOX4, which mediates the activation of NOX4, especially the mitochondrial translocation. Further exploration of the molecular basis behind this interesting finding could provides insight into the communication between the two ROS-generating systems and the differences in the redox homeostasis regulatory mechanisms of tumor cells compared to normal cells.

Our study found that Neu-p11 leads to caspase-1-dependent cell death, cell pyroptosis, in chordoma cells through activation of NLRP3 inflammasomes. NLRP3 is a member of a family of intracellular conserved pattern recognition receptors that can be activated by a variety of intracellular "damaging stimuli" such as ROS and intracellular DNA. The classic downstream pathway after NLRP3 inflammasome activation is IL-1 $\beta$ maturation with Caspase-1, which ultimately leads to inflammation[36]. The role of NLRP3 in the fate determination of tumor cells has been less reported. We found that NOX4 caused damage to the mitochondrial of chordoma cells, increased accumulation 
bioRxiv preprint doi: https://doi.org/10.1101/2020.02.12.946665; this version posted February 13, 2020. The copyright holder for this preprint (which was not certified by peer review) is the author/funder. All rights reserved. No reuse allowed without permission.

of mAROS and increased mitochondrial permeability, and finally activated NLRP3 jointly with intracytoplasmic mtDNA. These results suggest that activation of NLRP3 is theoretically feasible for anticancer. Further investigation of the regulatory differences between Caspase-1-mediated apoptosis and IL-1 $\beta$ maturation after inflammasome activation will be key to understanding the anticancer mechanism of NLRP3. 


\section{Conclusions}

We found that neu-p11 causes oxidative damage of mitochondria by activating NOX4 and promoting NOX4 mitochondrial translocation, thereby activating the NLRP3 inflammasome and eventually inducing Caspase-1-dependent pyroptosis in chordoma cells. This work provides experimental evidence for the clinical application of Neu-p11 against chordoma.

\section{Data Availability}

All relevant data are within the manuscript and figures.

\section{Conflicts of Interest}

The authors declare that the research was conducted in the absence of any commercial or financial relationships that could be construed as a potential conflict of interest.

\section{Funding Statement}

This work was supported by the "Qing Feng" Undergraduate Innovation and Entrepreneurship Scholarship of Shanghai Medical college, Fudan University (QF1703) to STZ. The fundershad no role in study design, data collection and analysis, decision to publish, or preparation of the manuscript.

\section{Acknowledgments}

We sincerely thank $\mathrm{Li} \mathrm{Hu}$ M.S. of Laboratory of Neuropharmacology and Neurotoxicology in Shanghai University for providing technical assistance. 


\section{Reference}

1. Brian P Walcott, Brian V Nahed, Ahmed Mohyeldin, et al. Chordoma: Current concepts, management, and future directions[J]. Lancet Oncology, 2012, 13(2):e69-76. 2. Mcmaster M L , Goldstein A M , Bromley C M , et al. Chordoma: Incidence and Survival Patterns in the United States, 1973-1995[J]. Cancer Causes \& Control, 2001, 12(1):1-11.

3. Barry J J , Jian B J , Sughrue M E, et al. The Next Step: Innovative Molecular Targeted Therapies for Treatment of Intracranial Chordoma Patients[J]. Neurosurgery(1):1.

4. Vande Walle, Lieselotte, Lamkanfi, Mohamed. Pyroptosis[J]. Current Biology, 26(13):R568-R572.

5. Bergsbaken, Tessa, Fink, Susan L, Cookson, Brad T. Pyroptosis: host cell death and inflammation[J]. Nature Reviews Microbiology, 7(2):99-109.

6. Derangère, V, Chevriaux, A, Courtaut, F, et al. Liver X receptor $\beta$ activation induces pyroptosis of human and murine colon cancer cells[J]. Cell Death \& Differentiation, 21(12):1914-1924.

7. Alexander Wree, Akiko Eguchi, Matthew D. McGeough, et al. NLRP3 inflammasome activation results in hepatocyte pyroptosis, liver inflammation, and fibrosis in mice $[\mathrm{J}]$. Hepatology, 2014, 59.

8. Tschopp, Jurg, Schroder, Kate SLLR3 inflammasome activation: the convergence of multiple signalling pathways on ROS production?[J]. Nature Reviews Immunology, $10(3): 210-215$.

9. Zhou Rongbin, Yazdi, Amir S, Menu, Philippe, et al. A role for mitochondria in NLRP3 inflammasome activation[J]. Nature, 475(7354):122-122.

10. Ding, Z, Liu, S, Wang, $X$, et al. LOX-1, mtDNA damage, and NLRP3 inflammasome activation in macrophages: implications in atherogenesis[J]. Cardiovascular Research, 103(4):619-628.

11. Iyer, Shankar S, He, Qiong, Janczy, John R, et al. Mitochondrial Cardiolipin Is Required for Nlrp3 Inflammasome Activation[J]. Immunity, 39(2):311-323.

12. O’Neill, Luke A.J. Cardiolipin and the Nlrp3 Inflammasome[J]. Cell Metabolism, 18(5):610-612.

13. Edward Mills, Ping Wu, Dugald Seely, et al. Melatonin in the treatment of cancer: a systematic review of randomized controlled trials and meta-analysis[J]. Journal of Pineal Research, 2005, 39(4):360-366.

14. D E Blask, LA Sauer, R T Dauchy, et al. Melatonin inhibition of cancer growth in vivo involves suppression of tumor fatty acid metabolism via melatonin receptormediated signal transduction events[J]. Cancer Research, 1999, 59(18):4693-4701.

15. Soumya Nair, Sandra Suresh, Arya Kaniyassery, et al. A review on melatonin action as therapeutic agent in cancer[J]. Frontiers in Biology, 2018, 13(3).

16. Markus, Regina P, Ferreira, Zulma S, Fernandes, Pedro A.C.M, et al. The ImmunePineal Axis: A Shuttle between Endocrine and Paracrine Melatonin Sources[J]. Neuroimmunomodulation, 14(3-4):126-133. 
17. Srinivasan, V, Spence, D W, Pandi-Perumal, S. R, et al. Therapeutic Actions of Melatonin in Cancer: Possible Mechanisms[J]. Integrative Cancer Therapies, 7(3):189203.

18. Liu, L, Wang, T, Yang, X, et al. MTNR1B loss promotes chordoma recurrence by abrogating melatonin-mediated $\beta$-catenin signaling repression. J Pineal Res. 2019; 67:e12588.

19. Zhu, Ping, Liu, Jianfeng, Shi, Jinxin, et al. Melatonin protects ADSCs from ROS and enhances their therapeutic potency in a rat model of myocardial infarction[J]. Journal of Cellular \& Molecular Medicine:n/a-n/a.

20. Espino, Javier, Rodríguez, Ana B, Pariente, José A. Melatonin and Oxídative Stress in the Diabetic State: Clinical Implications and Potential Therapeutic Applications[J]. Current Medicinal Chemistry, 2019.

21. Hong-Mei Zhang, Yiqiang Zhang, Bin-Xian Zhang. The role of mitochondrial complex III in melatonin-induced ROS production in eultured mesangial cells[J]. Journal of Pineal Research, 2010, 50(1):78-82.

22. Liu Rui, Wang Hui-li, Deng Man-jing, et al. Melatonin Inhibits Reactive Oxygen Species-Driven Proliferation, Epithelial-Mesenchymal Transition, and Vasculogenic Mimicry in Oral Cancer[J]. Oxidative Medicine and Cellular Longevity, 2018:1-13.

23. Castroviejo, Darío Acuña, López, Luis C, Escames, Germaine, et al. Melatoninmitochondria Interplay in Health Cand Disease[J]. Current Topics in Medicinal Chemistry, 2011, 11(2):--

24. Dario Acuna-Castroviejo, Miguel Martin, Manuel Macias, et al. Melatonin, mitochondria, and cellular bioenergetics[J]. Journal of Pineal Research, 2001, 30(2):6574.

25. Meihua She, Xiaojian Deng, Zhenyu Guo, et al. NEU-P11, a novel melatonin agonist, inhibits weight gain and improves insulin sensitivity in high-fat/high-sucrosefed rats $[\mathrm{J}]$. Pharmacological Research the Official Journal of the Italian Pharmacological Society, 59(4):248-253.

26. Yu, Jianwu, Wei, Jing, Ji, Ling, et al. Exploration on Mechanism of a New Type of Melatonin Receptor Agonist Neu-p11 in Hypoxia-Reoxygenation Injury of Myocardial Cells[J]. Cell Biochemistry \& Biophysics, 70(2):999-1003.

27. Joris Messens, Nicolas Rouhier, Jean-François Collet. Redox Homeostasis[M]// Oxidative Stress and Redox Regulation. 2013.

28. Christophe Blanchetot, Johannes Boonstra. The ROS-NOX connection in cancer and angiogenesis[J]. Crit Rev Eukaryot Gene Expr, 2008, 18(1):35-45.

29. Tohru Kamata. Roles of Nox1 and other Nox isoforms in cancer development[J]. Cancer Science, 2009, 100.

30. Wiechmann A F, Vrieze M J, Dighe R K, et al. Melatonin Directly Influences Rod Photoreceptor Sensitivity to Light via a Specific Melatonin Receptor: A Transgenic Xenopus laevis Model[J]. 2003.

31. Barberino R S, Menezes V G, Ribeiro A E A S, et al. Melatonin protects against cisplatin-induced ovarian damage in mice via the MT1 receptor and antioxidant activity[J]. 2017:1244-1255. 
32. Mor M, Spadoni G, Diamantini G, et al. Antioxidant and Cytoprotective Activity of Indole Derivatives Related to Melatonin[J]. 2003, 527:567.

33. Patio, Paloma, Parada, Esther, Farré-Alins, Victor, et al. Melatonin protects against oxygen and glucose deprivation by decreasing extracellular glutamate and Nox-derived ROS in rat hippocampal slices[J]. Neurotoxicology, 57:61-68.

34. Sánchez-Sánchez, Ana M, Martín, Vanesa, García-Santos, Guillermo, et al. Intracellular redox state as determinant for melatonin antiproliferative vs cytotoxic effects in cancer cells[J]. Free Radical Research, 45(11-12):1333-1341.

35. David E. Blask, Sean T. Wilson. Melatonin Action on Human Breast Cancer Cells: Involvement of Glutathione Metabolism and the Redox Environment[M]. Springer US, 1995.

36. Yazdi, Amir S, Guarda, et al. Nanoparticles activate the NLR pyrin domain containing 3 (Nlrp3) inflammasome and cause pulmonary inflammation through release of IL-1 \{alpha and IL-1 \{beta [Immunology][J]. 2010, 107(45):19449-19454.

37. Bai J, Cervantes C, Liu J, et. al. DsbA-L prevents obesity-induced inflammation and insulin resistance by suppressing the mtDNA release-activated EAS-cGAMPSTING pathway. Proc Natl Acad Sci U S A. 2017 Noy 14;114(46):12196-12201. 
Figures

A

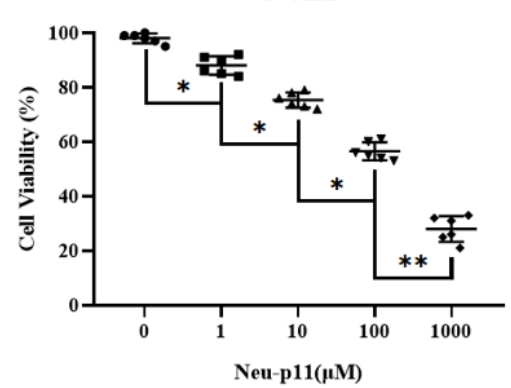

C

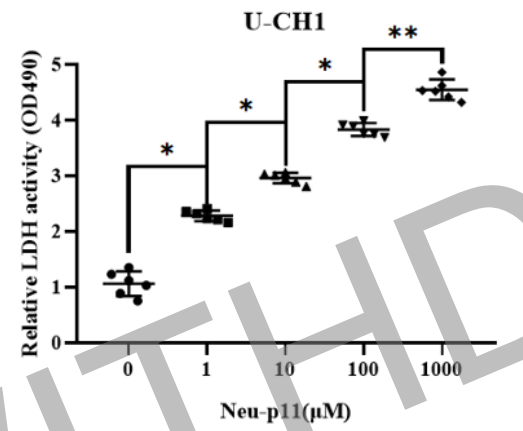

B

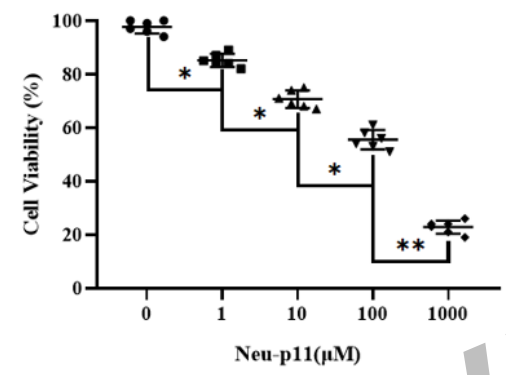

D

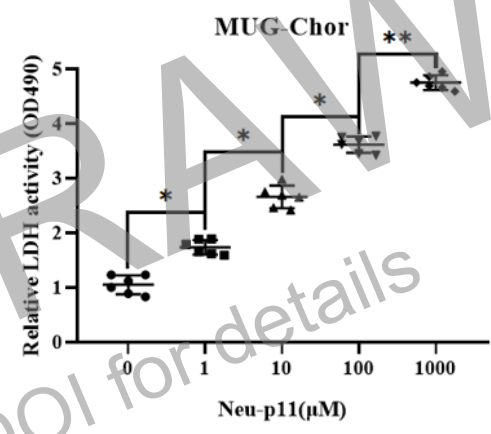

E

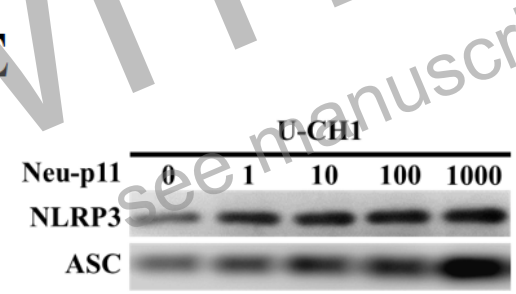

pro-Caspase-1

Cleaved-Caspase-1

$$
\text { pro-IL-1 } \beta
$$

$\beta$-actin

$\mathbf{F}$

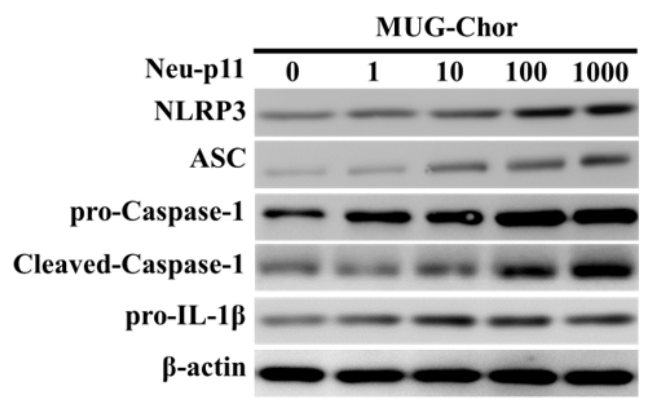

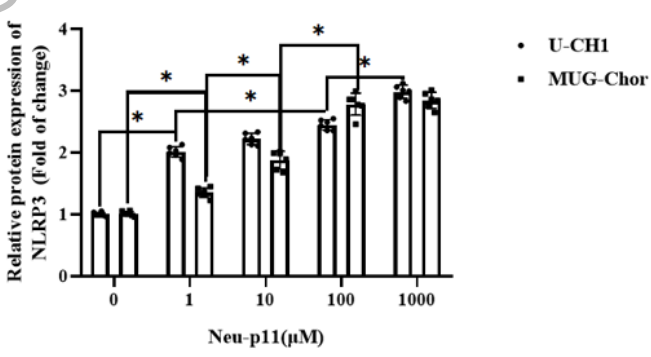

$\mathbf{H}$

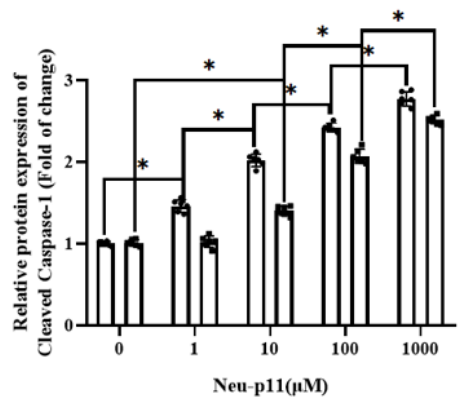

- U-CH1

- MUG-Chor

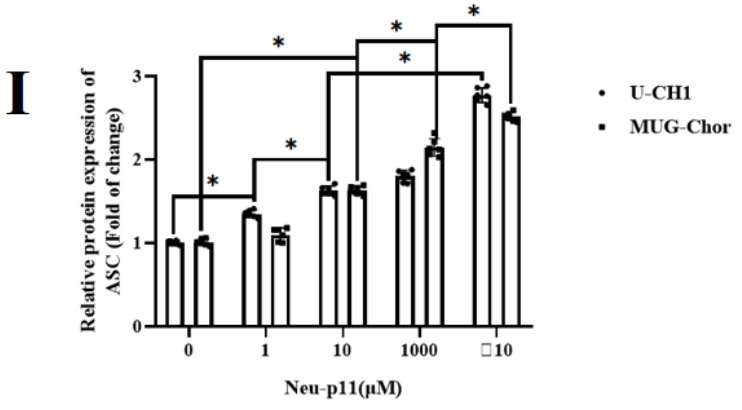

Fig.1 Neu-p11 inhibits chordoma cell growth and activates NLRP3 inflammasone.

(A-D) CCK-8 assay and LDH activity assay were used to evaluate the effect of Neup11 on chordoma cell proliferation and viability. Neu-p11 inhibited the growth of 
bioRxiv preprint doi: https://doi.org/10.1101/2020.02.12.946665; this version posted February 13, 2020. The copyright holder for this preprint (which was not certified by peer review) is the author/funder. All rights reserved. No reuse allowed without permission.

chordoma cells and had obvious cytotoxic effect, and the effect was concentrationdependent.

(F-G) Western blot analysis of the expression of NLRP3 inflammasome in Neu-p11treated chordoma cells. Neu-p11 activates NLRP3 inflammasome, and the expression of each subunit (NLRP3, ASC, pro-Caspase-1, pro-IL-1 $\beta$ ) increases with the increase of neu-p11 concentration. Neu-p11 increases the expression of cleaved-Caspase-1. Data in (A), (B), (C), (D), (G), (H) and (I) are presented as mean \pm SEM. $\mathrm{n}=5, * P$ 0.05 . 
A

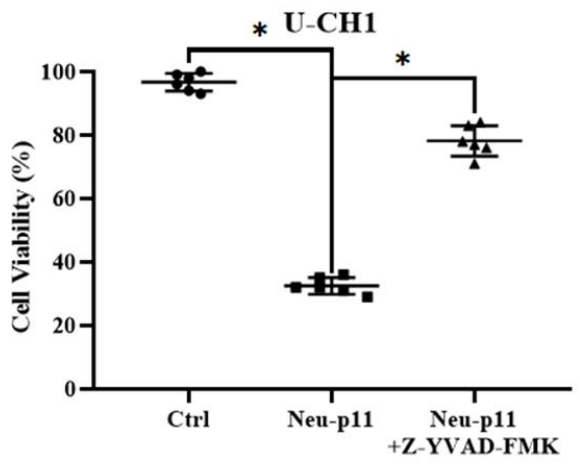

$\mathbf{C}$

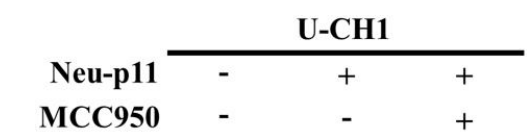

pro-Caspase-1

Cleaved-Caspase-1

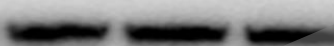

\section{$=$}

$$
\text { PARP }
$$

$\beta$-actin

$\mathbf{E}$

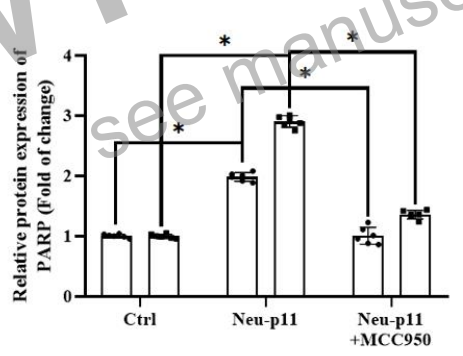

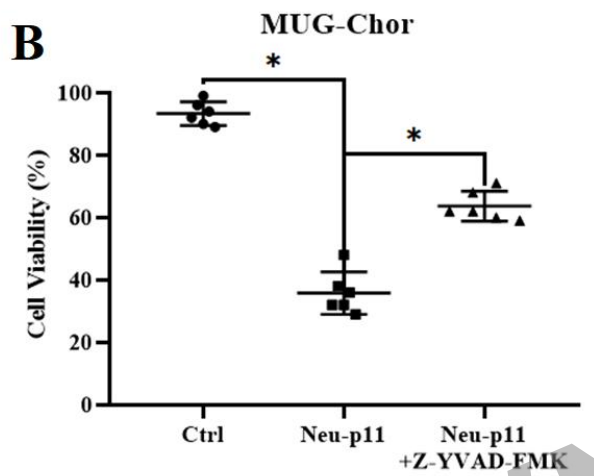

D

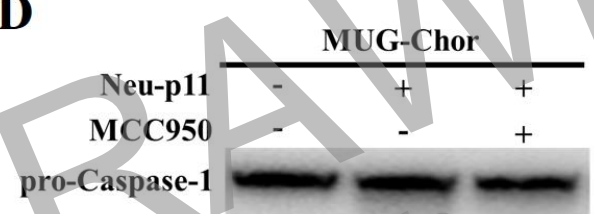

Cleaved-Caspase-1

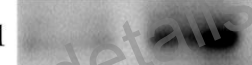

PÁRP

$\beta$-actin

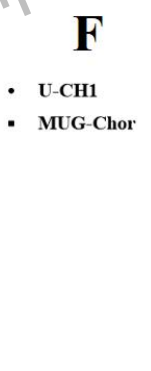

- U-CH1

- MUG-Chor

Fig.2 Neu-p11 induced chordoma cell pyroptosis depend on NLRP3 activation.

(A-B) The caspase-1 specific inhibitor Z-YVAD-FMK() significantly blocked the antiproliferation effect of Neu-p11 on chordoma cells.

(C-D) The NLRP3 specific inhibitor MCC950() attenuated Caspase-1 maturation on Neu-p11-treated chordoma cells. MCC950 attenuated the increased expression of proCaspase-1, Cleaved-Caspase-1, and PARP in Neu-p11-treated chordoma cells. (E-F) MCC950()blocked the anti-proliferation effect of Neu-p11 on chordoma cells.

Data in (A), (B), (E) and (F) are presented as mean \pm SEM. $\mathrm{n}=5, * P<0.05$. 
A

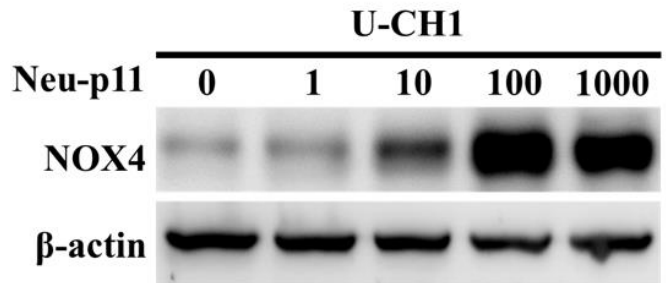

B

\begin{tabular}{|c|c|c|c|c|c|}
\hline \multirow[b]{2}{*}{ Neu-p11 } & \multicolumn{5}{|c|}{ MUG-Chor } \\
\hline & 0 & 1 & 10 & 100 & 1000 \\
\hline NOX4 & Exis & 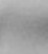 & ns: & $=0$ & wes \\
\hline
\end{tabular}

C

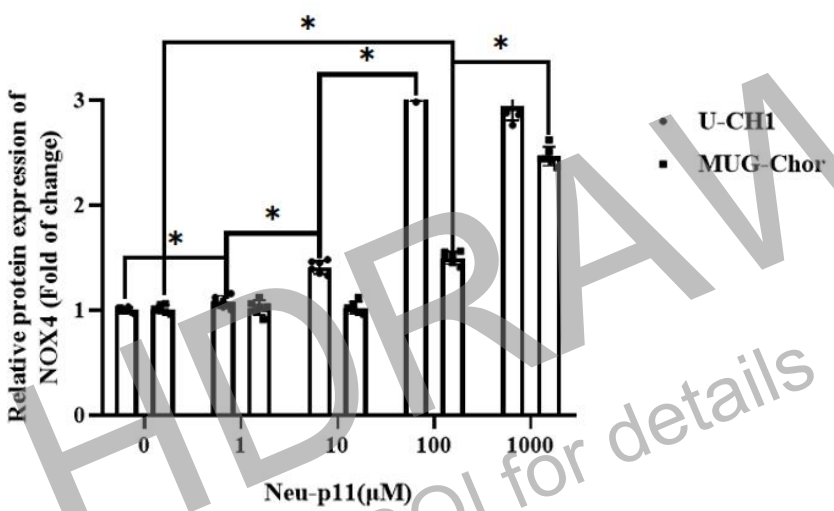

Fig.3 Neu-p11 induced NOX4 activation on chordoma cells.

(A-C) Western blot analysis of the expression of NOX4 in Neu-p11-treated chordoma cells. Neu-p11 increased NOX4 expression and the effect was dose-dependent.

Data in $(C)$ are presented as mean \pm SEM. $\mathrm{n}=5, * P<0.05$. 
A

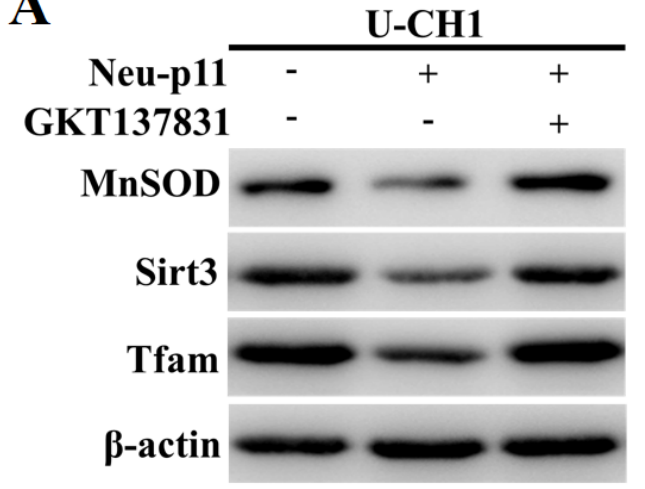

B
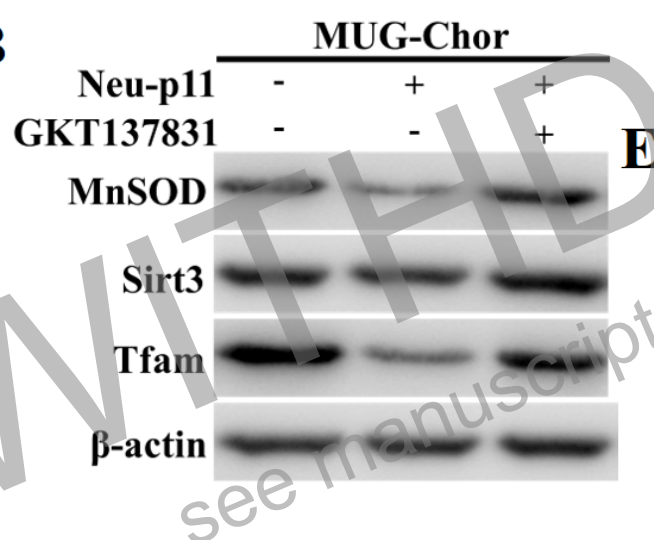

C

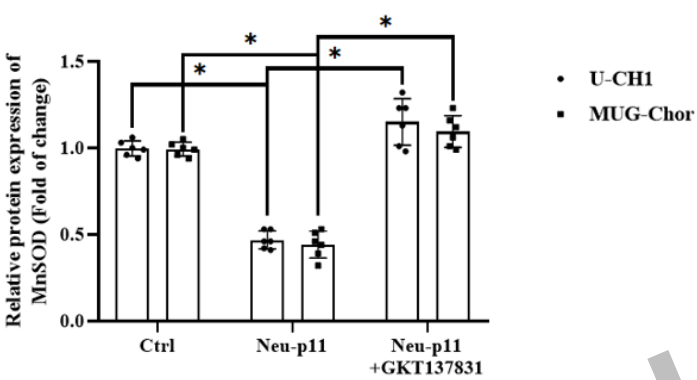

D

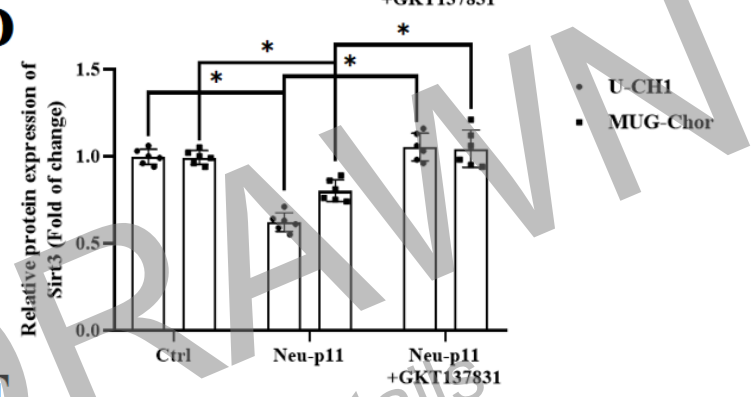

E

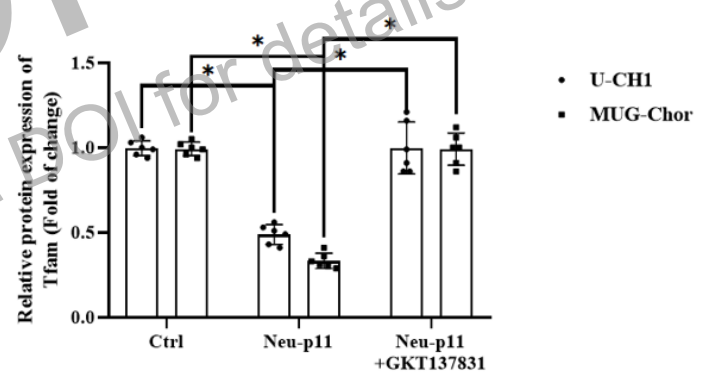

Fig.4 Neu-p11 damaged mitochondrial anti-oxidation ability depend on NOX4 activation.

(A-E) Western blot analysis showed that the expressions of mitochondrial antioxidant proteins (MnSOD, Sirt3) and mitochondrial DNA replication factor (Tfam) were decreased. Nox4 specific inhibitor GKT137831 inhibited the effect of Neu-p11 on mitochondrial anti-oxidation ability.

Data in (C), (D) and (E) are presented as mean \pm SEM. $\mathrm{n}=5, * P<0.05$. 
A

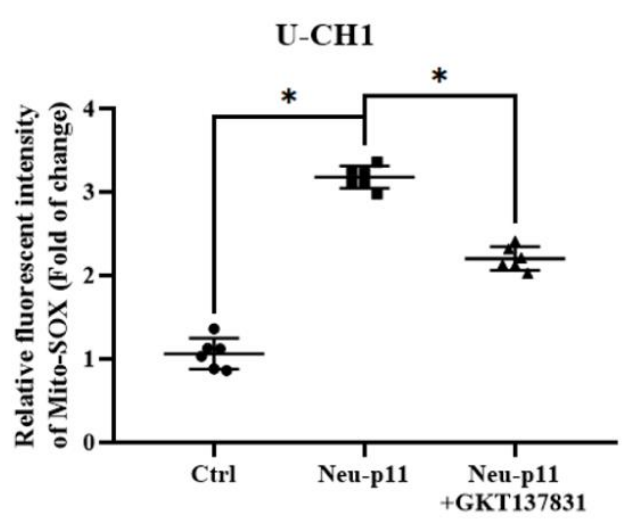

B

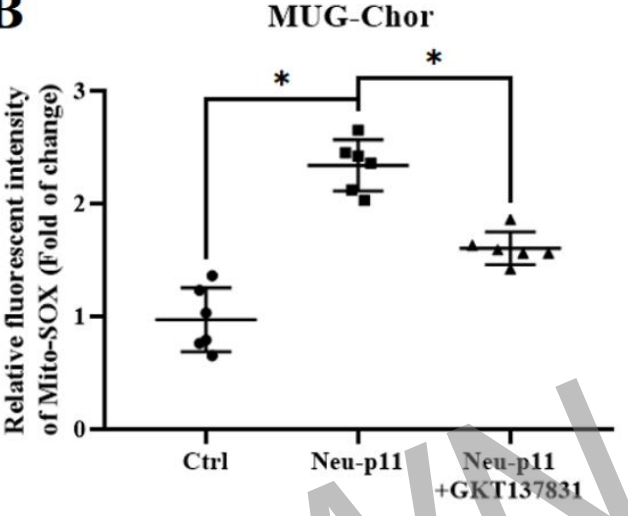

C

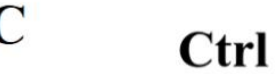

Neu-p11

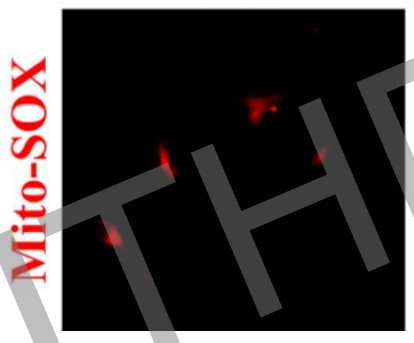

Neu-p11

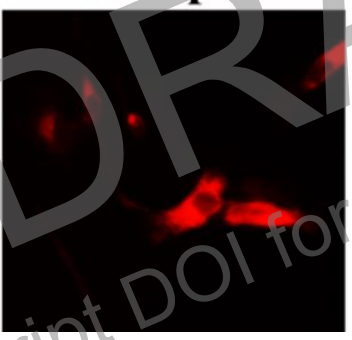

+GKT137831

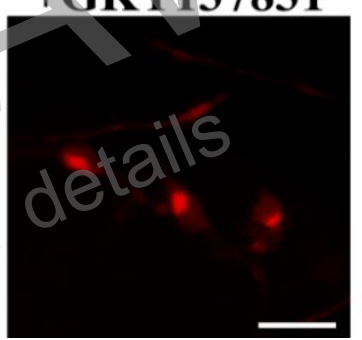

Fig.5 Neu-p11 damaged mitochondrial anti-oxidation ability depend on NOX4 activation.

(A-B) Mito-SOX (Mitochondrial ROS indicator) staining indicated that Neu-p11 led to a significant increase in mitochondria derived ROS (mtROS). Nox4 specific inhibitor GKT137831 attenuated the increase of mtROS.

(C) Representative image of Mito-SOX staining on U-CH1 cells.

Scale bar $=30 \mu \mathrm{m}$ in $(\mathrm{C})$. Data in $(\mathrm{A})$ and $(\mathrm{B})$ are presented as mean \pm SEM. $\mathrm{n}=6, * P$ $<0.05$. 
A

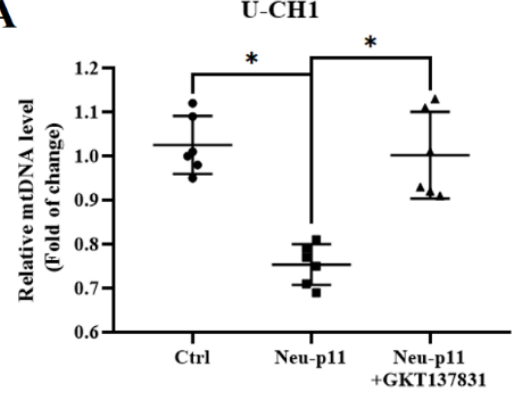

C

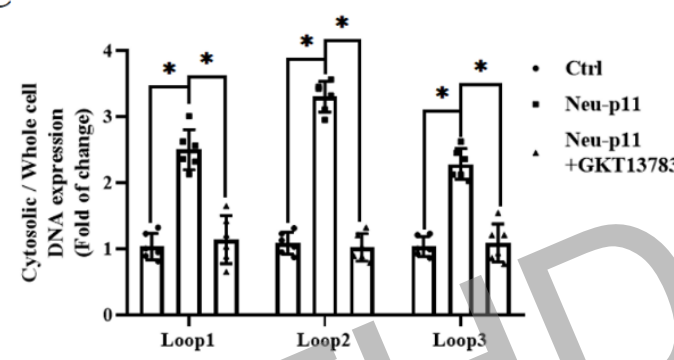

$\mathbf{E}$

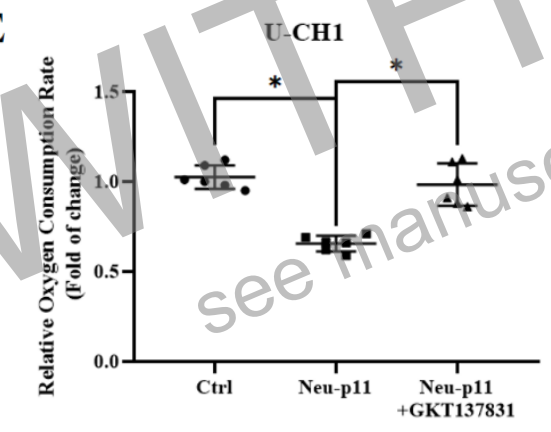

B

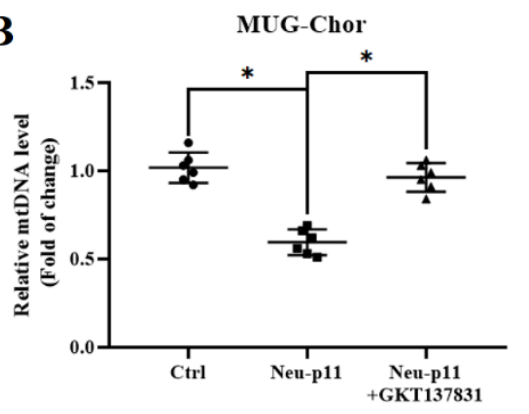

D

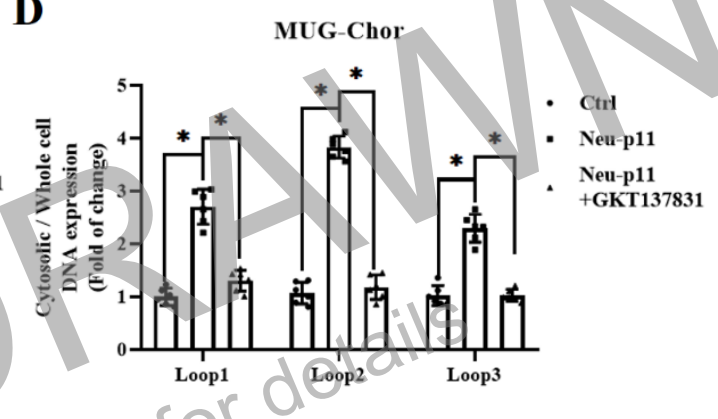

F

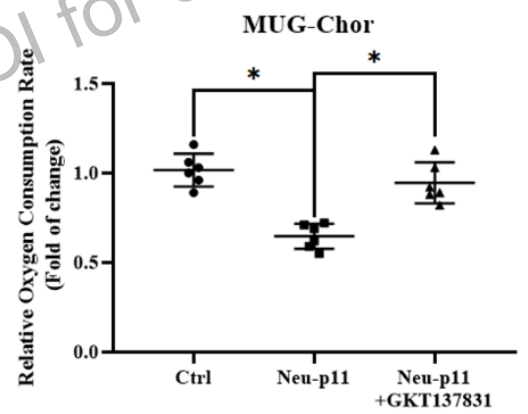

Fig.6 Neu-p11 caused DNA damage and increased permeability of mitochondria depend on NOX4 activation.

(A-D) PCR showed that Neu-p11 resulted in decreased copy number of mitochondrial DNA and increased cytoplasmic DNA, indicating increased mitochondrial permeability and mitochondrial DNA damage. Nox4 specific inhibitor GKT137831 attenuated the DNA damage and increased permeability of mitochondria.

(E-F) Cell oxygen consumption detection found that Neu-p11 resulted in decreased mitochondrial oxygen consumption, indicating that mitochondrial respiratory chain was damaged.

Data in (A), (B), (C), (D), (E) and (F) are presented as mean \pm SEM. $\mathrm{n}=5, * P<0.05$. 


\section{A}

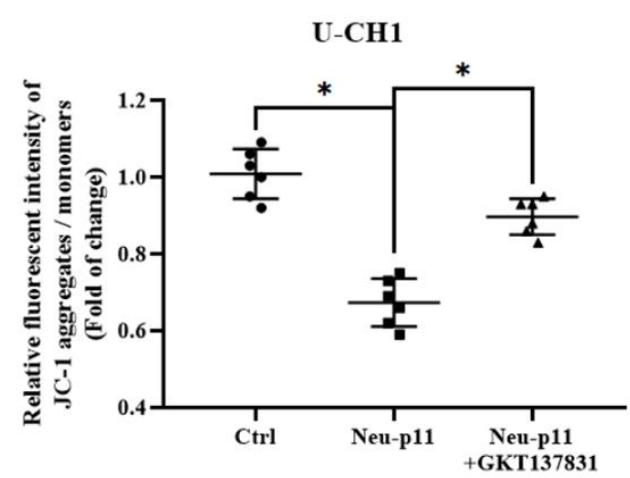

B

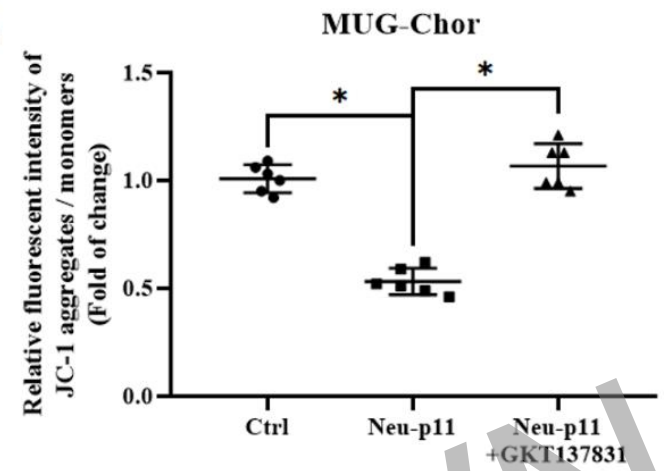

C

JC-1 aggregates

JC-1 monomers
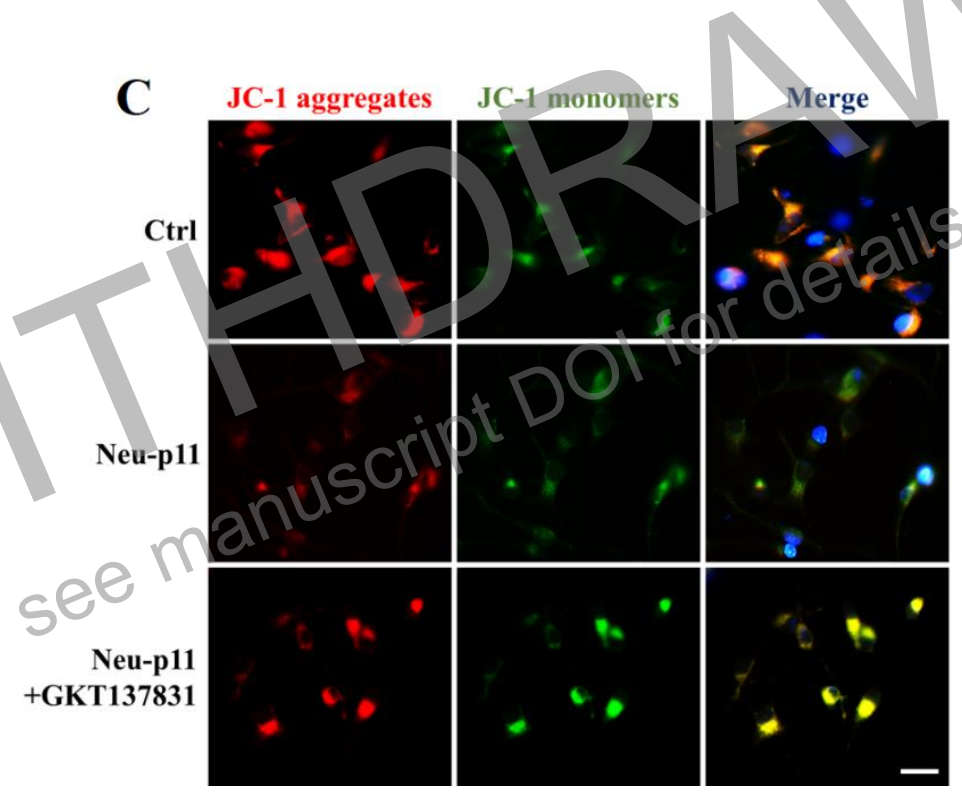

Fig.7 Neu-p11 reduced mitochondrial membrane potential depend on NOX4 activation.

(A-B) JC-1 (mitochondrial membrane potential probe) staining showed that neu-p11 decreased mitochondrial membrane potential. Nox4 specific inhibitor GKT137831 attenuated mitochondrial membrane potential reduction on Neu-p11-treated chordoma cells.

(C) Representative image of JC-1 staining on U-CH1 cells.

Scale bar $=50 \mu \mathrm{m}$ in (C). Data in (A) and (B) are presented as mean \pm SEM. $\mathrm{n}=6, * P$ $<0.05$. 


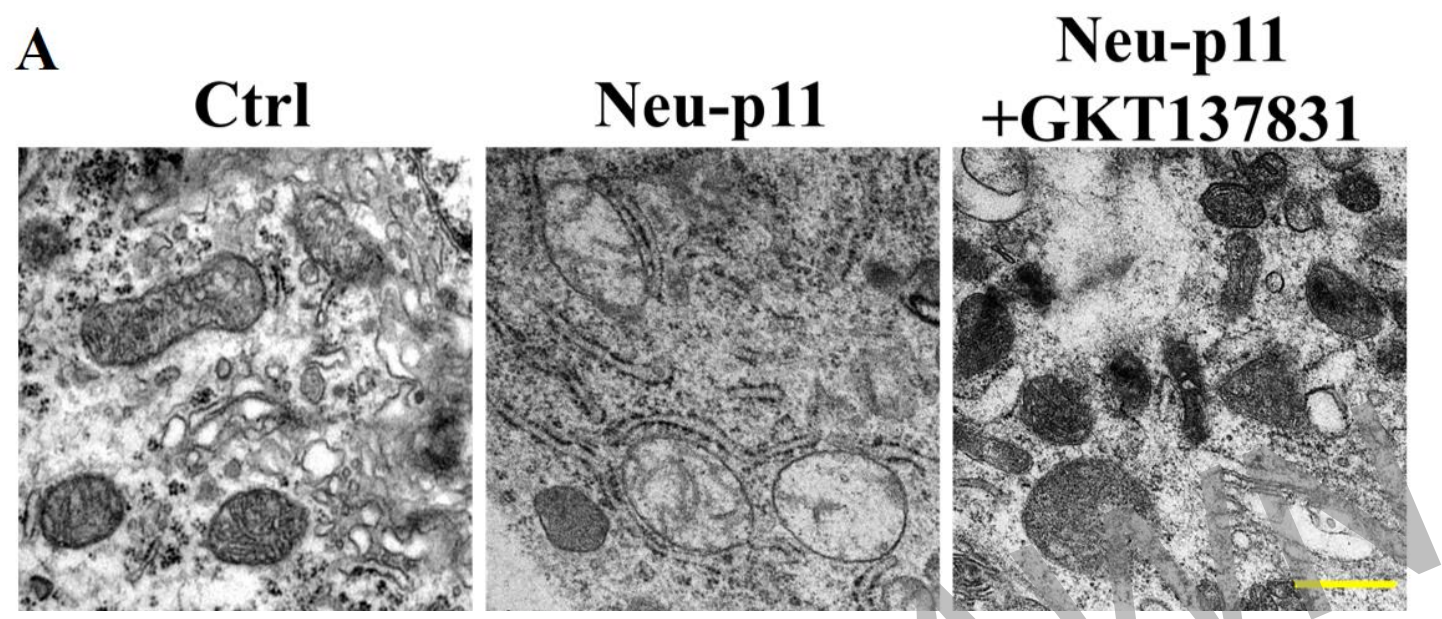

Fig.8 Neu-p11 induced mitochondrial structure damage depend on NOX4 activation.

(A) Transmission electron microscopy (TEM) confirmed that the mitochondria in the neu-p11 treatment chordoma cells were swollen, the matrix density was decreased, and the mitochondrial crest was disturbed and disappeared. Nox4 specific inhibitor GKT137831 reduced mitochondrial structure damage.

Scale bar $=2 \mu \mathrm{m}$ in $(A)$. 
A

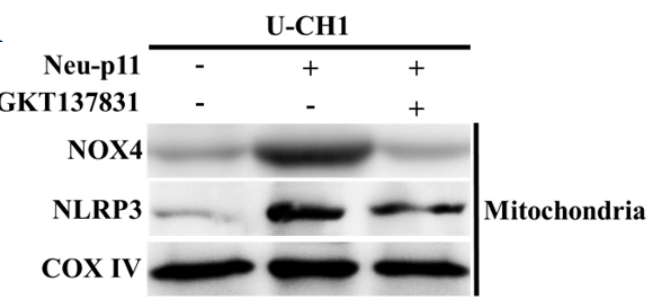

C
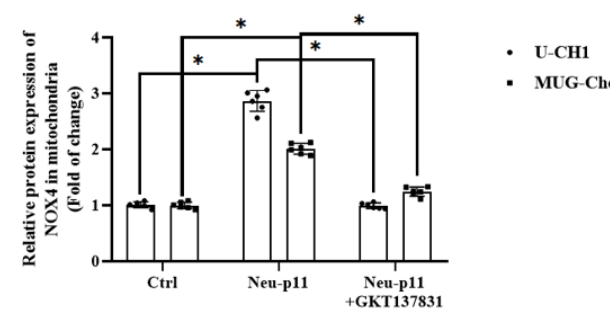

- MUG-Chor
B

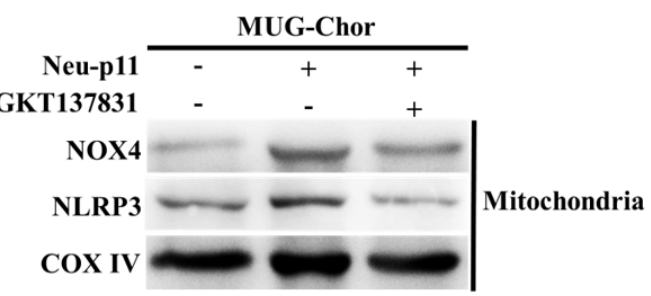

D

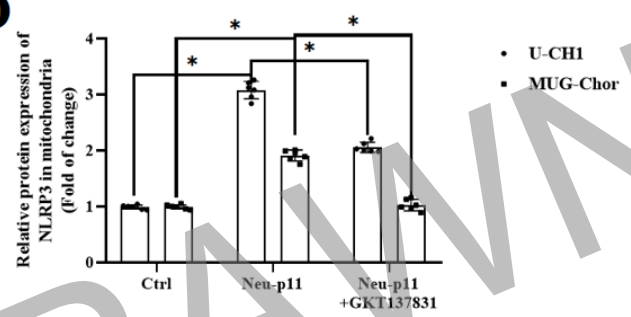

Fig.9 GKT137831 inhibited NOX4 translocation to mitochondria and the contact between NLRP3 and mitochondria in Neu-p11-treated chordoma cells.

(A-D) Western blot analysis of the expression of NOX4 and NLRP3 in mitochondrial in different groups. Neu-p11 increased NOX4 translocation to mitochondria and the contact between NLRP3 and mitochondria. GKT137831 inhibited the effect of Neup11.

Data in (C) and (D) are presented as mean \pm SEM. $\mathrm{n}=5, * P<0.05$. 


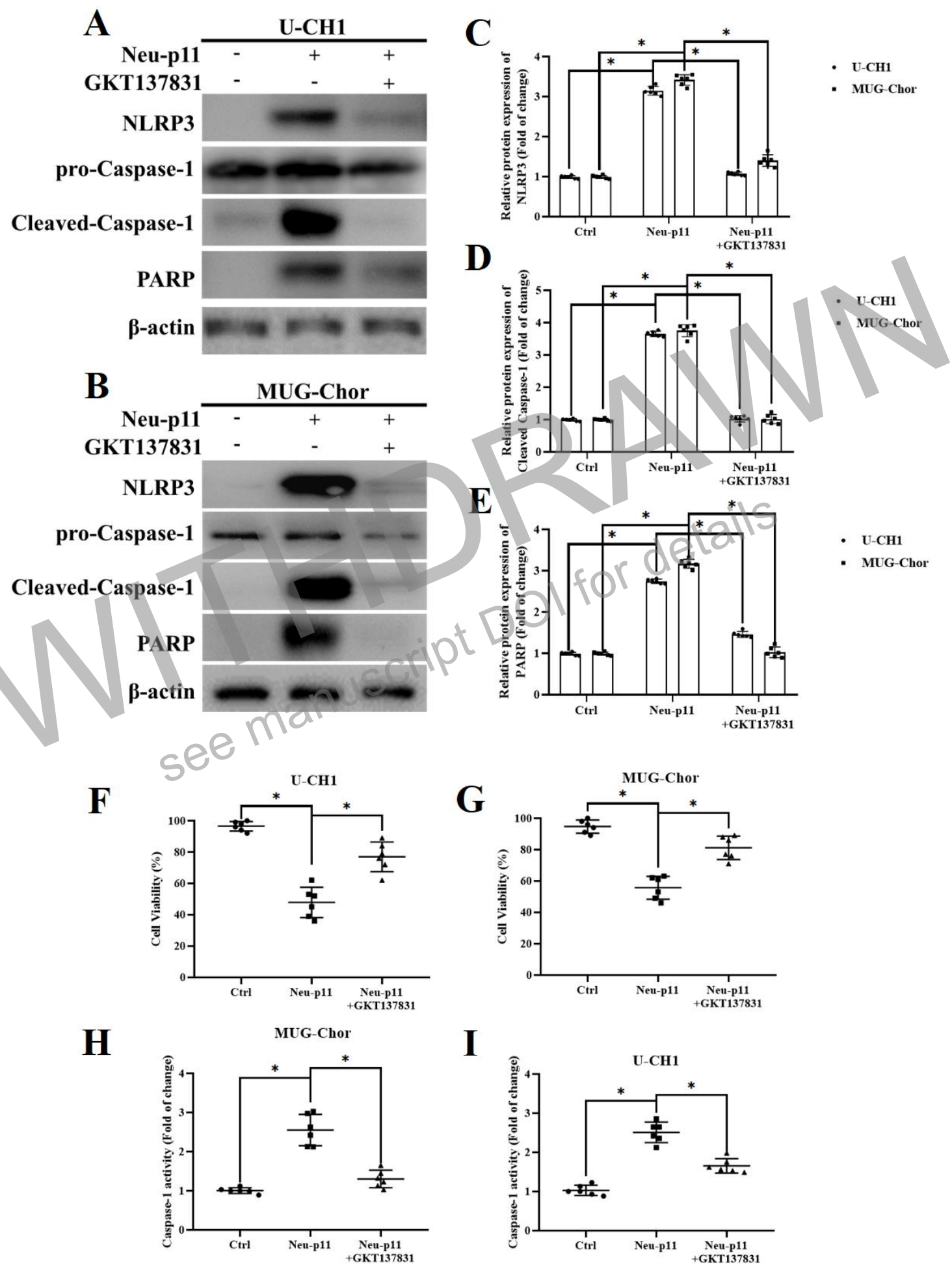

Fig.10 GKT137831 attenuated the activation of inflammatory body, Caspase-1 activation and increased PARP expression of chordoma cells caused by Neu-p11, and inhibit the cytotoxic effect of Neu-p11 on chordoma.

(A-E) Western blot analysis of the expression of NLRP3, pro-Caspase-1, CleavedCaspase-1 and PARP in different groups. Neu-p11 increased the activation of 
bioRxiv preprint doi: https://doi.org/10.1101/2020.02.12.946665; this version posted February 13, 2020. The copyright holder for this reprint (which was not certified by peer review) is the author/funder. All rights reserved. No reuse allowed without permission.

inflammatory body, Caspase-1 activation and increased PARP expression of chordoma cells. GKT137831 inhibited the effect of Neu-p11.

(F-G) GKT137831 inhibit the cytotoxic effect of Neu-p11 on chordoma.

(H-I) GKT137831 inhibit the increased Caspase-1 activity of Neu-p11 on chordoma.

Data in (C), (D), (E), (F), (G), (H) and (I) are presented as mean \pm SEM. $\mathrm{n}=5, * P<$ 0.05 . 